\title{
«Erst kommt das Fressen ...
}

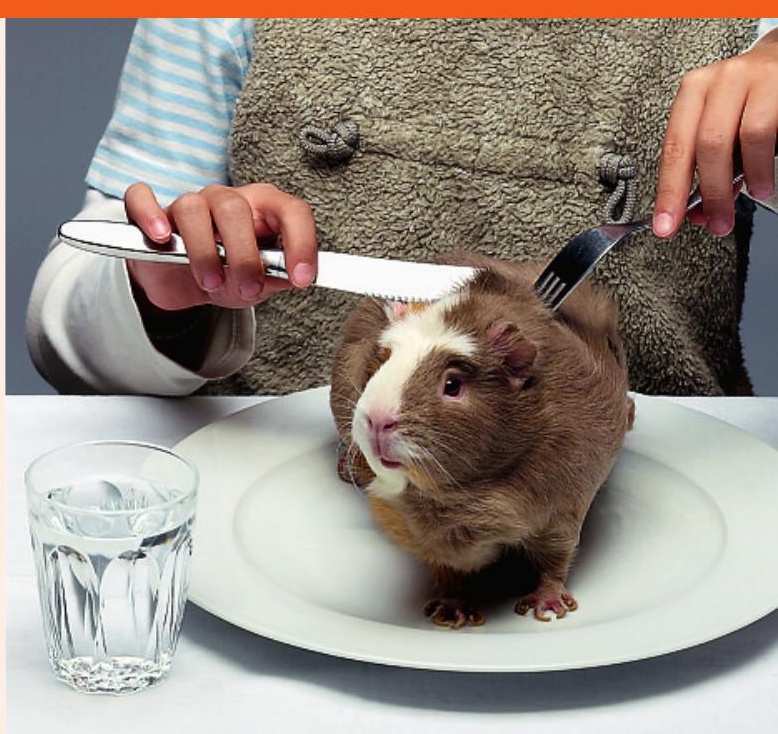

Erhard Taverna

* Essen und Macht - Eine Ausstellung übers Schlemmen, Schlachten und Hungern. Mühlerama,

bis 27. September 2009, Zürich-Tiefenbrunnen. www.muehlerama.ch.

Öffnungszeiten

Di-Sa 14-17 Uhr, So 10-17 Uhr.
... dann kommt die Moral - erst muss es möglich sein auch armen Leuten - vom grossen Brotlaib sich ihr Teil zu schneiden», singt die Seeräuberbraut Jenny in Bertold Brechts «Dreigroschenoper».

Um «Essen und Macht» geht es auch in der «Ausstellung übers Schlemmen, Schlachten und Hungern» im Mühlerama in Zürich*. Im Backsteinschloss der Belle Epoque, wo früher Bier gebraut wurde, liefert die heutige Schaumühle in Tiefenbrunnen ausser einigen Tonnen Mehl im Jahr auch ganzjährige Informationen zum Getreideanbau und ergänzende Sonderausstellungen rund um Ernährung, Ess- und Trinkkultur. Dass Essen viel mit Moral und Macht zu tun hat, erfahren Besucherinnen und Besucher auf einem abwechslungsreichen Rundgang vom Schlachthof zur Fürstentafel und vom Welthandel zur Verpflegung im Gefängnis, begleitet von kurzen Abstechern zu Hungersnöten, Hungerkünstlern und Essstörungen. Zur Vertiefung einzelner Ausstellungsthemen laden im Laufe der nächsten Monate Kurzführungen und Gespräche ein, zu Themen wie Macht und Ohnmacht am Familientisch, Schwein gegen Prestige, Nahrungstabus und Kannibalismus, wenn Essen Sünde wird oder Welthandel und Hungerproblematik. Wo es immer gut nach Gemahlenem und Gebackenem riecht, werden auch die Kinder an besonderen Anlässen mit leckeren Zugaben verwöhnt.

Die beschränkten Mittel erlauben nur eine Vogelschau auf das weite Feld von Essen und Macht. Dennoch ist es erstaunlich, was die Macher an Einstiegsmöglichkeiten zusammengestellt haben. Das beginnt mit unserer Macht über die Nutztiere, die im Akkord auf der Schlachtbank enden. Im Jahr 2007 wurden in der Schweiz 465000 Tonnen Fleisch verzehrt, was ohne Massentierhaltung nicht möglich wäre. Dazu kamen noch 56000 Tonnen Fische und Meerestiere aus den Kühltanks der Fabrikschiffe und Fangflotten. Mit zunehmendem Wohlstand steigt weltweit die Nachfrage nach Fleisch. Vom Wasserverbrauch ist leider nicht die Rede. Zum Beispiel von den 4600 Litern für jeden CH-Einwohner täglich, deren grösster Teil für die Herstellung von Lebensmitteln verbraucht wird. Allein für die Herstellung eines Kilogramms Rindfleisch sind 15000 Liter Wasser nötig! Das Schicksal der Tiere wiederholt sich im Stundenbuch der Catharina von Cleves um 1440, denn die verdammten Seelen verenden in der Hölle in brodelnden Kochtöpfen und auf feurigen Rosten. Das Gemetzel ist einseitig. Schosstiere kommen nicht auf den Teller und Menschenfleisch nur symbolisch als Hostie, die sich gemäss Konzil von 1225 in den Leib Christi verwandelt. Im opulenten Schmausen fürstlicher Gesellschaften erreichen die Fressorgien einen vorläufigen Höhepunkt. Aus der Riesenpastete steigt der Hofzwerg in den Kranz der gebratenen Pfauen im Federkleid. Wie im Gabenstreit des indianischen Potlatch, definiert das kulinarische Spektakel Rang, Hierarchie und soziale Identität. Wer spendet, kann auch nehmen, am Familientisch oder in der grossen Politik. Viele Hungersnöte waren absichtlich herbeigeführte Massenmorde. Wie verdrängte Erinnerungen nennen die nebeneinander aufgereihten Pultklappen auf der abgewandten Innenseite die Ukraine 1932/33, Leningrad 
1941-44, Chinas «grossen Sprung» von 1959-61 und Biafra 1967. Alle stehen für absichtlich herbeigeführte oder fahrlässig verursachte Katastrophen mit vielen Millionen Hungertoten. Wohl erwähnt, aber leider wenig ausgeführt, sind die Hinweise auf den globalen Handel mit Nahrungsmitteln und unsere vollständige Abhängigkeit von deren Futterkrippen. Soja aus Brasilien für $\mathrm{CH}$-Schweine, Mais und Zuckerrohr für volle Benzintanks, Subventionen für einheimische Zuckerrüben statt Zucker aus Entwicklungsländern zu einem fairen Preis oder EU-Produkte zu Schleuderpreisen auf den Märkten der Ärmsten, das sind einige wenige Beispiele für den Machtmissbrauch der Regierungen und Nahrungsmittelkonzerne. Seit den 70er Jahren sind biologisch hergestellte Produkte im Aufwind. 18 verschiedene Label hängen wie Schlüsselanhänger an der Stellwand. Von Bio Knospe bis Fairtrade Max Havelaar orientiert die Rückseite über Anwendung und Bewertung der viel zu vielen Gütesiegel.

Auch die Ablehnung von Essen verleiht Macht. Fastenheilige und Wundermädchen stan- den im Mittelalter hoch im Kurs. Wo aber das Fasten weder ein sakraler Heilsweg noch die übermässige Gefrässigkeit bewundertes Kennzeichen eines hohen Standes ist, da schlägt die Stunde der Hungerkünstler und Lichtnahrungsmenschen. Die globale Asymmetrie von Überfressen und Zwangshungern produziert die Essstörungen der Anorektiker und Bulimiker. Wenn Disziplin und Selbstkontrolle vorherrschen, ist die stolze Selbstverneinung der Magersucht eine Insel der Reinheit. Oder sie führt zum Gegenextrem, wenn die geforderte Zurückhaltung in eine pathologische Fresslust umschlägt. Vom industriell erzeugten Überfluss an Nahrungsmitteln führt ein technisch-logischer Weg zur bariatrischen Chirurgie. Die Ausstellung zitiert einen ethischen Grundsatz des Weltärztebundes, der allen Medizinern die Beteiligung an Zwangsernährungen verbietet, und erinnert damit an Hungerstreiks und Gegenmassnahmen. Zahlreichere weitere Details belohnen den Betrachter mit Wissensstoff und weiteren Bezügen, die zum nachträglichen Vertiefen verlocken.

\section{Pädiatrie am Hôpital Albert-Schweitzer, Haiti}

Naoko König

Korrespondenz:

Naoko König

Busenhardstrasse 24

8704 Herrliberg

naokokoenig@hotmail.com
Im Frühling 2006 arbeitete ich während zweier Monate als Assistenzärztin in der Pädiatrieabteilung des Hôpital Albert-Schweitzer (HAS) von Deschapelles, Haiti.

\section{Zum ersten Mal in Haiti}

Vor meinem Aufenthalt in Haiti hatte ich nur vage Vorstellungen über die dortigen Verhältnisse. Medienberichte, aber auch Schilderungen von «Haiti-Erfahrenen» ergaben ein überwiegend düsteres Bild: Von extremer Armut und politischen Unruhen, hoher Arbeitslosigkeit und Analphabetismus war die Rede. Haiti sei nicht nur gezeichnet durch den Umstand, dass seine Bevölkerung während zweier Jahrhunderte unter verschiedenen diktatorischen Regimes zu leiden hatte und politisch wie auch sozial völlig heruntergewirtschaftet worden sei. Auch ökologisch sei das Land ausgebeutet worden und werde infolge kompletter Abholzung regelmässig durch Überschwemmungen und Erdrutsche heimgesucht. In diesem Land werde Voodoo praktiziert, und mitten in der Karibik, von beliebten Feriendestinationen umgeben, sei doch alles anders, hoffnungslos.

Einige der genannten Probleme, mit denen Haiti zu kämpfen hat, wurden während meines Aufenthaltes deutlich sichtbar, so insbesondere

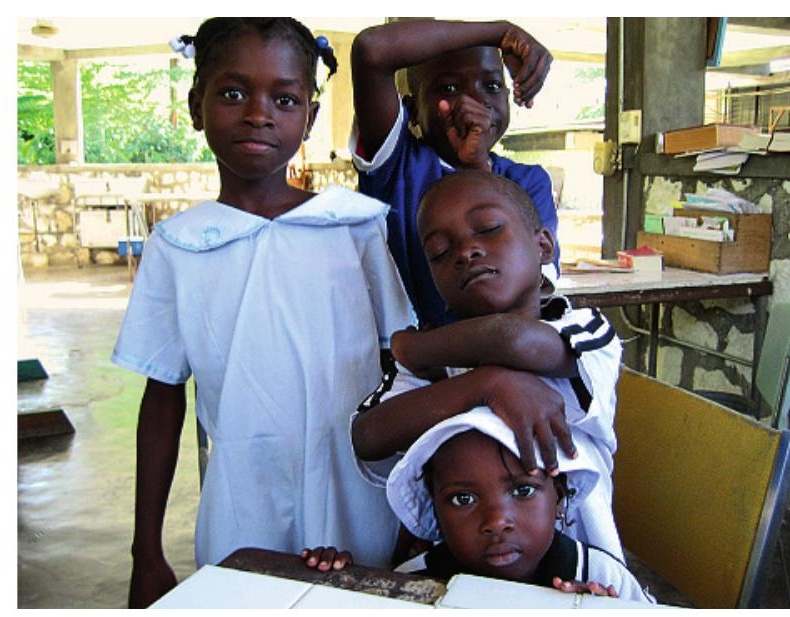

Neugierige Patienten und ihre Geschwister.

die grosse Armut und die Folgeschäden der Abholzung. Eindrücklich war auch der Respekt, den die Bevölkerung den Voodoo-Priestern und ihren Ritualen zollte. Die politische Situation war trotz der Parlamentswahlen, die gerade stattfanden, einigermassen stabil. Obwohl die Lage oberflächlich betrachtet ruhig und friedlich wirkte, war stets eine unterschwellige Angst vor erneuten Umstürzen, aber auch eine grosse Vorsicht - etwa bei der Äusserung politischer Ansichten - zu spüren. Gleichzeitig fand ich bei den Haitianern eine 


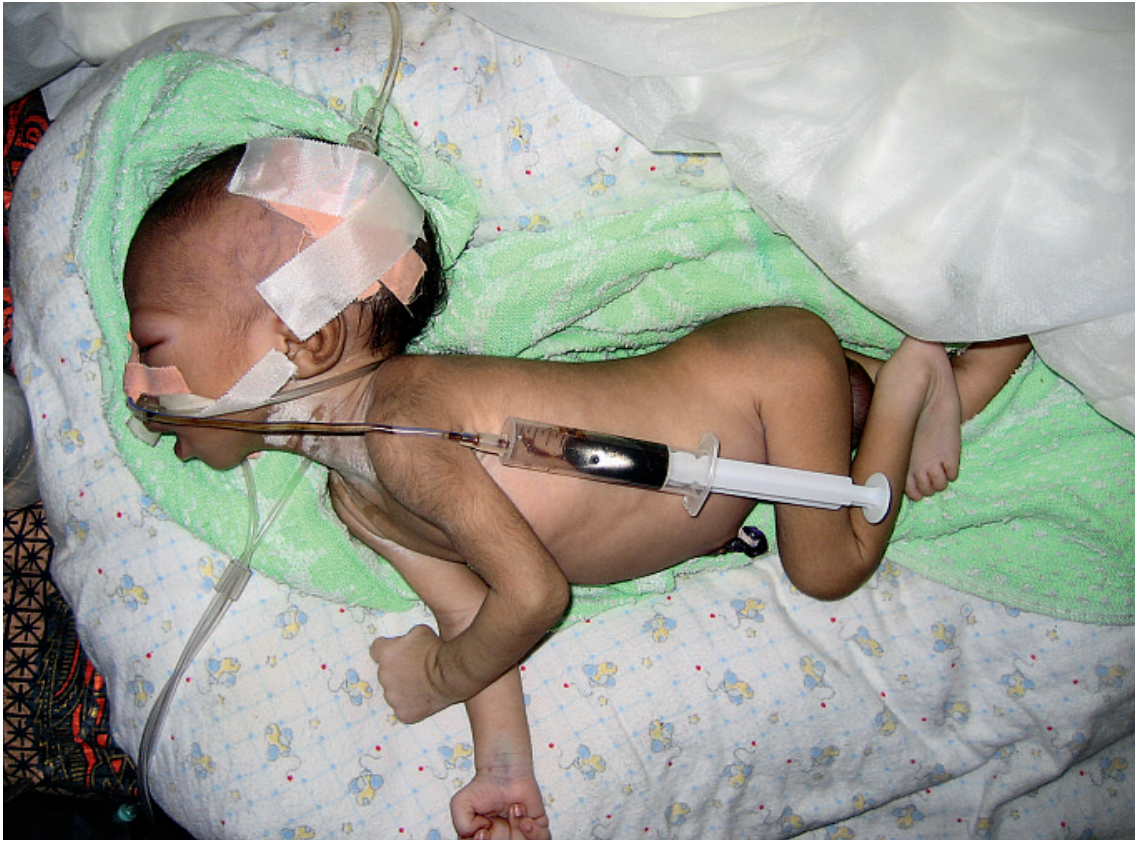

Mangelernährtes Frühgeborenes mit neonataler Sepsis.

Kraft, selbst in elenden und ziemlich aussichtslosen Situationen weiter zu funktionieren, um zu überleben. Diese Art Optimismus hat sich wohl in all den schweren Zeiten herausgebildet.

Die Arbeit in der Pädiatrie war für mich sehr spannend und bereichernd, vor allem weil ich vielen in der Schweiz nicht verbreiteten Krankheitsbildern begegnete, die ich nur aus Lehrbüchern und Vorlesungen kannte. Der Kontakt mit den Patienten und vor allem mit den Eltern war nicht immer einfach: Zu den sprachlichen Schwierigkeiten kamen ganz banale, kulturell bedingte Kommunikationsprobleme hinzu. Beispielsweise waren sich viele Haitianer nicht gewohnt, auf eine konkrete Frage eine präzise Antwort zu geben. Gerade für die Menschen aus den Bergen ist wahrscheinlich allein die Umgebung des Spitals, der Kontakt mit «Blancs» neu und erschreckend.

\section{Häufige Probleme in der Pädiatrie}

Für mich am schockierendsten waren die vielen unterernährten Kinder, die wir behandeln mussten. Diese Kinder, die meisten mit Kwashiorkor (Eiweissmangelernährung), waren schwer krank. Nebst der Schwächung durch Mangelernährung an sich waren sie auch stark gefährdet für Infektionen, selbst eine «einfache» Magen-Darm-Grippe konnte für diese Kinder tödlich sein. Erschwerend bei der Behandlung war, dass einige dieser Kinder an HIV und/oder Tuberkulose erkrankt waren. Oft wurden die Kinder in einem sehr schlechten $\mathrm{Zu}$ stand ins Spital gebracht, so dass wir mit unserer Behandlung zu spät kamen. Eindrücklich war für mich auch, wie sehr die Kinder nicht nur physisch, sondern auch psychisch angeschlagen waren. Ohne Ausnahme sassen sie während Tagen, Wochen, manchmal auch Monaten mit traurigem Gesichtsausdruck in ihren Betten. Es dauerte oft lange, bis sie wieder von selbst zu trinken und zu essen begannen. Sogar dafür war die Kraft nicht ausreichend - die Kinder waren voll damit beschäftigt zu überleben. Die meisten hätten als gesunde Kinder aufwachsen und gedeihen können, wenn sie nur genug zu essen bekommen hätten. Dass in Haiti, nur eineinhalb Flugstunden von Miami entfernt, Kinder verhungern müssen, fand ich äusserst traurig. Sich dieser Realitäten und der beschränkten eigenen Möglichkeiten bewusst zu sein und dennoch die Hoffnung nicht zu verlieren, ist etwas, das ich in Deschapelles zu lernen begonnen habe.

Neben Mangelernährung, HIV und Tuberkulose sah ich viele Patienten mit verschiedenen infektiösen Erkrankungen, u.a. - wie erwähnt auch Erkrankungen, die man in der Schweiz nur aus Büchern kennt. Hier waren die direkten Auswirkungen der fehlenden medizinischen Grundversorgung zu spüren. In der Umgebung des HAS werden sogenannte Dispensaires unterhalten, mit denen der Bevölkerung Zugang zu medizinischer Grundversorgung vermittelt wird. Unter anderem werden in den Dispensaires Impfprogramme durchgeführt. Bei «Out district»-Patienten, die nicht in Dispensaires versorgt wurden, gab es zum Beispiel Fälle von Starrkrampf wegen fehlender Impfung. Starrkrampf ist eine sehr schwere Erkrankung, die oft tödlich verläuft.

\section{Knappe Ressourcen}

Ich fand es schwierig, in bestimmten Fällen einfach nichts tun zu können, in denen ich wusste, dass man in unserer betreffend die medizinischen Möglichkeiten «entwickelteren» Welt viel hätte bewirken können. Beispielsweise diagnostizierten wir bei einem 9jährigen Knaben eine Form einer Leukämie, bei der mit Chemotherapie 90\% der betroffenen Kinder geheilt werden können. In diesem Fall konnten wir nicht mehr tun, als den Eltern zu empfehlen, nach Port-au-Prince zu gehen, wo Chemotherapien durchgeführt werden. Uns war klar, dass ein solcher Vorschlag mit höchster Wahrscheinlichkeit aus finanziellen Gründen nicht befolgt werden kann.

Die stark eingeschränkten oder gar nicht vorhandenen finanziellen Ressourcen des Spitals und der Patienten lehrten mich, neue Prioritäten in der Diagnostik und Therapie zu setzen. Wenn ich zum Beispiel bei einem Kind mit Verdacht auf Lungenentzündung in der Schweiz vor einer Behandlung mit Antibiotika zur Bestätigung eine Röntgenuntersuchung durchgeführt hätte, 


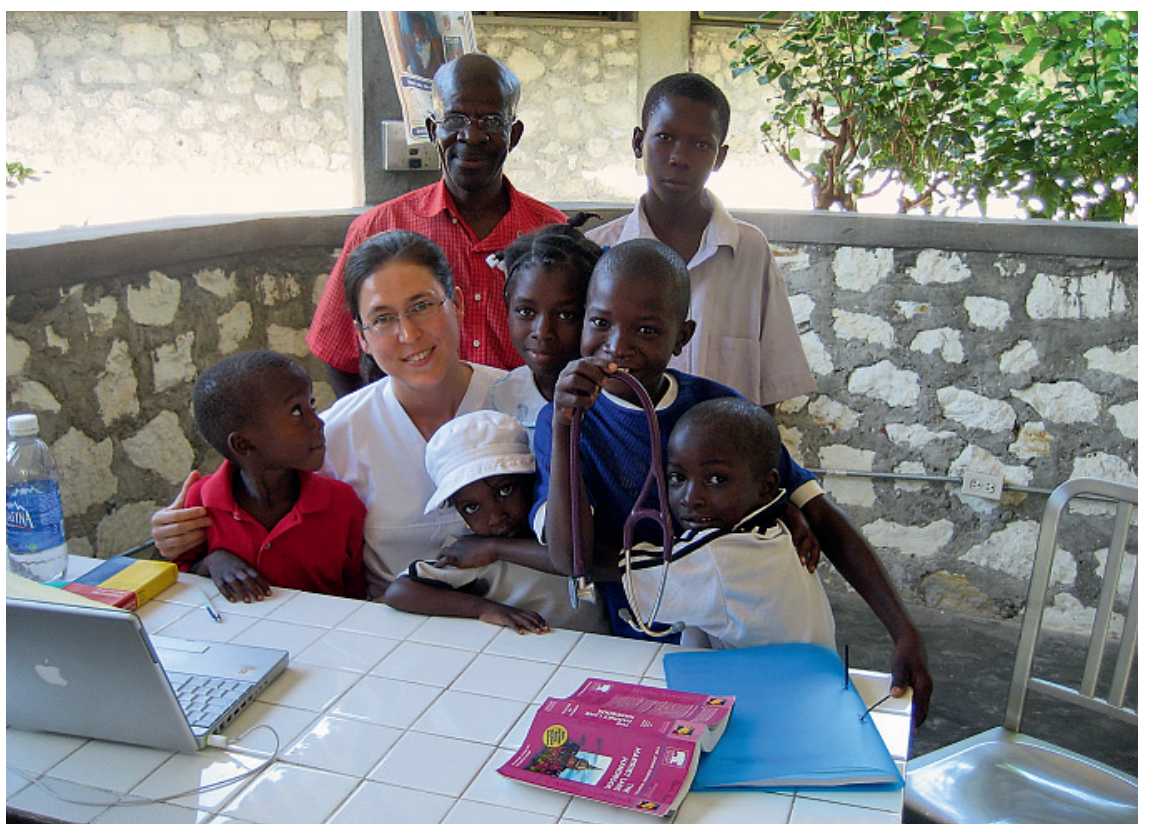

Im Einsatz im pädiatrischen Ambulatorium.

musste ich hier entscheiden, ob ich auf eine solche Untersuchung verzichten und statt dessen mit dem wenigen Geld, das zur Verfügung stand, direkt mit einer Behandlung beginnen sollte.

Es war indes ermutigend zu sehen, dass mit wenigen Mitteln viel erreicht werden kann. Beispiele dafür sind die vorne genannten Auswirkungen der Impfprogramme oder die rasche Besserung nach intravenöser Flüssigkeitsgabe bei einem stark dehydrierten Kind mit Brechdurchfall.

\section{Umstrukturierung am HAS}

Seit 2004 arbeitet die Spitalleitung an einer Umstrukturierung zur Erhaltung des Spitals, zur Verbesserung der Zusammenarbeit innerhalb des Spitals, aber auch zum Ausbau und zur Dezentralisation der medizinischen Grundversorgung in den Dispensaires. Im Rahmen der Umstrukturierung wurde die Bettenzahl reduziert und Personal entlassen. Eingeführt wurden zudem Be- handlungskosten für die Patienten. Eine weitere Massnahme war beispielsweise die Einführung dreisprachiger morgendlicher Rapporte und Fortbildungen. Diese werden nunmehr auf englisch, französisch und kreolisch durchgeführt.

Auch wenn die ergriffenen Massnahmen teilweise zu einer gewissen Verunsicherung hinsichtlich der Zukunft des HAS geführt haben, sind sie wie mir scheint - insgesamt positiv aufgenommen worden und hatten deutliche Verbesserungen zur Folge. So führte die Mehrsprachigkeit der morgendlichen Rapporte und Fortbildungen insbesondere zu einer deutlich verbesserten Motivation und Teilnahme der haitianischen Mitarbeiter.

Die offene Haltung der Spitalleitung bezüglich der Probleme des Spitals und die kontinuierliche Ermutigung zur Optimierung der Zusammenarbeit spiegelten sich im Alltag wider. Der Kontakt zwischen Ärzten und anderen Spitalarbeitern war im Allgemeinen sehr gut.

\section{Zukunft}

Den Haitianern und besonders den Kindern in Haiti ist zu wünschen, dass ihr Land irgendwann doch eine politische Stabilität und Schritte in Richtung wirtschaftliches Wachstum erreicht, damit für alle Kinder der Zugang zu medizinischer Versorgung, zum Schulbesuch und einer Berufsausbildung mit entsprechend besseren Perspektiven möglich wird. Solange ein grosser Teil der Kinder nicht einmal genug Nahrung, geschweige denn Ausbildung in der Schule bekommen kann, liegt dieses Ziel noch in weiter Ferne.

Für mich selbst wünsche ich, dass ich mit mehr Berufserfahrung und mehr Möglichkeiten, den Menschen in Haiti zu helfen, nach Deschapelles zurückkehren kann. Die Zeit am HAS war für mich trotz all der Schwierigkeiten, denen ich begegnete, bereichernd und faszinierend. Die Freunde, die ich in Deschapelles gefunden habe, begleiten mich in meinem Alltag. Mesi ampil (danke) für die schöne Zeit! 